\title{
A Spectroscopic and Photometric Survey for Pre-Main Sequence Binaries
}

Eike W. Guenther

Thüringer Landessternwarte Tautenburg, 07778 Tautenburg, Germany

Viki Joergens, Ralph Neuhäuser,

MPI für Extraterrestrische Physik, 85740 Garching, Germany

Guillermo Torres,

Harvard-Smithsonian Center for Astrophysics, 60 Garden Street, Cambridge, MA 02138, USA

Natalie Stout Batalha,

Planetary Systems Branch NASA Ames Research Center Mailstop 245-3 Moffett Field, CA 94035-1000, USA

Jyotsna Vijapurkar,

Homi Bhabha Centre for Science Education, V. N. Purav Marg, Mankhurd, Mumbai 400088, India

Matilde Fernández,

Instituto de Astrofísica de Andalucia, Apdo 300418080 Granada, Spain

Reinhard Mundt

MPI für Astronomie, Königstuhl 17, 69117 Heidelberg, Germany

\begin{abstract}
We give here an overview of the current state of our survey for pre-main sequence spectroscopic binaries. Up to now we have taken 739 spectra of 250 pre-main sequence stars. We find that $8 \%$ of the stars show significant radial velocity variations, and are thus most likely spectroscopic binaries. In addition to the targets showing radial velocity variations, $6 \%$ of the targets are double-lined spectroscopic binaries i.e., the total fraction of spectroscopic binaries is expected to be about 14\%. All short-period SB2s are monitored photometrically in order to search for eclipses. An eclipsing SB2 would allow the direct measurement of the masses of both stellar components. Measurements of the stellar masses together with determinations of the stellar radii are a crucial test of evolutionary tracks of pre-main sequence stars.
\end{abstract}




\section{Introduction}

The most fundamental parameter of a star is its mass, which determines almost everything about its birth, life, and death. For low mass pre-main sequence stars this parameter is practically always derived by comparing the location of the star in the Hertzsprung-Russell diagram with theoretically calculated evolutionary tracks, i.e. it has not been determined directly. Direct determinations of the mass are possible if the object is a double-line spectroscopic binary (SB2), and if additionally the inclination is known. The inclination can be determined either if the system is eclipsing, or if it is spatially resolved, or if the motion of the photocentre is measured astrometrically. In some special cases the inclination might also be estimated indirectly. For example, in the case of a circumbinary disk, it is reasonable to assume that the inclination of the orbit is roughly the same as the disk.

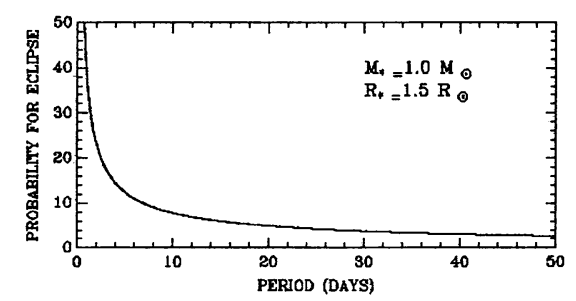

Figure 1. Probability for an eclipse to occur.

Up to now, all systems that have been spatially resolved have periods of more than 50 years, and are therefore not very suitable for determining the masses. With lunar occultations it is in principle possible to resolve systems with separations of 5 mas, bringing system with periods less than half a year into view. However, the problem is that only a few nearby star-forming regions are within the path of the moon. The situation will drastically improve in the near future when the VLTI becomes available, as the VLTI will allow us to resolve systems with a separation of 3 mas at $2.3 \mu \mathrm{m}$. Thus, the VLTI will be able to resolve systems of solar-mass pre-main sequence stars with periods down to 100 days or even less (distance $\leq 150 \mathrm{pc}$ ). The spectral-lines of such a system would be sufficiently well separated at periastron if the inclination is larger than about $45^{\circ}$, and $\mathrm{v} \sin \mathrm{i} \leq 20 \mathrm{kms}^{-1}$.

Hipparcos has also proven to be an extremely powerful tool for determining stellar masses (Martin et al. 1998). If the astrometric motion of the photocentre measured by Hipparcos is combined with the spectroscopic orbits, it is possible to determine the masses down to the brown dwarf regime (Zucker \& Mazeh 2000). Such work will be carried out with much higher accuracy and on much fainter stars when the astrometry satellite DIVA (Double Interferometer for Visual Astrometry) becomes available. DIVA will allow us to detect the astrometric motion of the photocentre of pre-main sequence binaries with periods longer than 20 days in nearby star-forming regions. The masses of all these binaries can then be derived, if this data is combined with the spectroscopic observations. Additionally, DIVA will eventually detect all pre-main sequence 
binaries with periods between 20 days and a few years in nearby star-forming regions if they are brighter than 16th magnitude in the visual.

But the precise measurement of the mass is also possible right away, namely for eclipsing SB2s since the analyses of the eclipse light curve provides a direct determination of their orbital inclination. As shown in Fig. 1 the probability for an eclipse to occur in a pre-main sequence binary system is more than $10 \%$ for binaries with periods less than 10 days and increasing for short-period systems.

\section{The Spectroscopic Survey}

As outlined above, the starting-point for the determination of the masses of pre-main sequence stars is the detection of spectroscopic binaries (SBs). We thus have initiated a survey for pre-main sequence SBs located in nearby starforming regions (Chamaeleon, Tucanae, Lupus, Scorpius-Centaurus, $\rho$ Oph, TW Hydrae, Taurus-Auriga, Corona Australis) using the high resolution Echelle spectrographs on the ESO 1.5m-telescope (FEROS), and on the 2m-telescope in Tautenburg. Up to now, we have obtained 739 spectra of 250 pre-main sequence stars.

In order to find SBs with periods as long as several hundred days, we intend to observe all 250 targets four times over a two year period. The spectroscopic binary candidates are then selected on the basis of the radial velocity (RV) variations. The limiting factor of the precision of the RV measurements is the stellar activity. For Weak-line T Tauri stars it is known that stellar activity leads to RV variations of a few $\mathrm{kms}^{-1}$ (Neuhäuser et al. 1998). We found that the same is also true for classical T Tauri stars. In order to be on the safe side, we thus consider only objects that show RV-variation of more than $10 \mathrm{kms}^{-1}$ as SB candidates (Fig. 2).

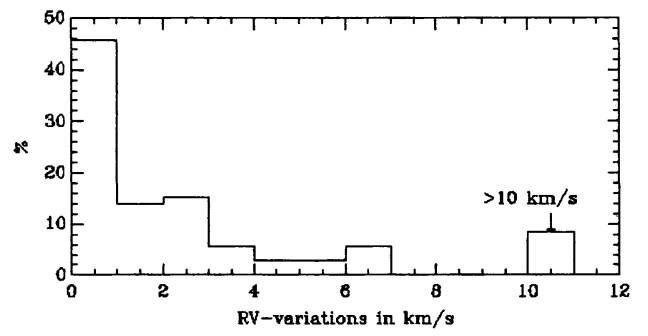

Figure 2. Distribution of RV variations. We find that $8 \%$ of the objects show RV variations that are larger than $10 \mathrm{kms}^{-1}$. They are considered to be good SB candidates.

In addition to these SB candidates, we monitor 16 SB2s. Of theses objects, eleven are entirely new, and five were known to be SBs but the orbits were unknown. Fig. 3 shows the spectra of RXJ1603.8-3938, were the orbit has recently been solved. The period of the system is $7.55574 \pm 0.00049$ days, $M_{A} \sin ^{3} i=0.2502 \pm 0.0088 M_{\odot}$ and $M_{B} \sin ^{3} i=0.2433 \pm 0.0136 M_{\odot}$. 


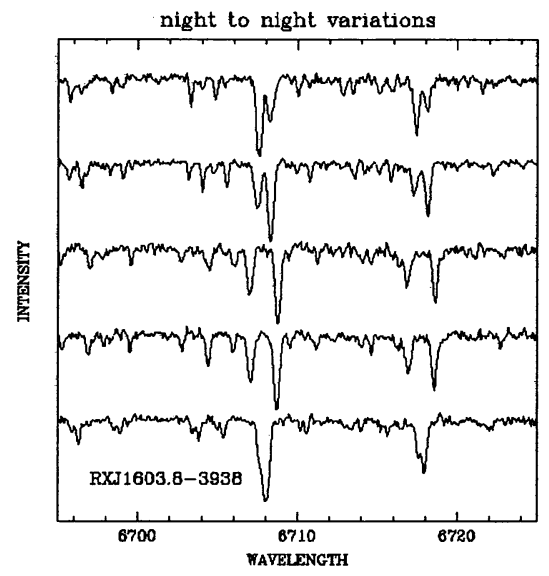

Figure 3. Small part of the spectra taken of RXJ1603.8-3938. Clearly visible are the two sets of lines of the two companions. The period of the system is about 7.6 days.

\section{The Photometric Survey}

Parallel to spectroscopic observations, we are monitoring the known as well as newly discovered short-period SB2s photometrically in order to detect eclipses. For systems with solved orbits we are able to predict the precise times of possible minima. For these systems we are performing a time-critical search for eclipses. The photometric observations are mostly carried out at the $0.8 \mathrm{~m}$ telescope on Wendelstein and the $1.23 \mathrm{~m}$ on Calar Alto. Beyond that, in April and May 2000, we undertook a large photometric campaign to monitor 6 of our most promising candidates in the southern hemisphere. In order to avoid nightlygaps we observed the targets using telescopes in Chile (1.5m Danish at La Silla), India $(1.0 \mathrm{~m}$ at the Vainu Bappu observatory) and Australia (40inch at Siding Springs). We obtained more than 1000 photometric images of our targets and the analyses of the data is underway.

Acknowledgments. We would like to thank the staff of La Silla, Siding Springs, Calar Alto, Wendelstein Observatory, the Vainu Bappu Observatory and Tautenburg for their help and assistance during our observing runs.

\section{References}

Armitage, P. J., \& Clarke, C. J. 1996, MNRAS, 280, 458

Martin, C., Mignard, F., Hartkopf, W. I., \& McAllister, H. A. 1998, ApJS, 133, 149

Neuhäuser, R., et al. 1998, A\&A, 334, 873

Zucker, S., \& Mazeh, T. 2000, AJ, 531, L67 